\title{
Auditory hallucinations in schizophrenia: helping patients to develop effective coping strategies
}

\author{
Douglas Turkington, Latoyah Lebert \& Helen Spencer
}

\begin{abstract}
SUMMARY
Auditory hallucinations (voices) are the most common symptom in schizophrenia, occurring in $70 \%$ of those with the diagnosis. A proportion respond to antipsychotic medication, but despite adequate concordance with prescribed medication, voices may still remain. Voice hearers often inadvertently activate coping strategies that maintain the symptoms and linked distress. Assessment can identify ineffective strategies. Effective strategies can then be initiated, starting with distraction to reduce distress in the short term and leading to focusing approaches that give an improved understanding of voice maintenance. This article describes how to recognise voice hearers' ineffective coping strategies and how to teach effective approaches that are a crucial catalyst in the recovery process.
\end{abstract}

\section{LEARNING OBJECTIVES}

- Recognise ineffective coping strategies used by patients who are distressed by auditory hallucinations in community and in-patient settings

- Understand how to teach patients distraction techniques and measure any benefit as an interim step

- Learn a variety of focusing approaches for delivery by mental health professionals and how these can be taught to patients in brief clinical sessions

\section{DECLARATION OF INTEREST}

D. T., L. L. and H. S. have received fees for delivering workshops on CBT

The purpose of this article is to reduce the distress caused by auditory hallucinatory experiences. Haddock et al (1996) undertook a randomised controlled trial of distraction versus focusing for voices and were able to demonstrate that patients could exert some control over voices, but that some strategies could have a negative impact on self-concept and esteem. In a further study (Haddock 1998) they found that any positive benefit of distraction approaches did not generalise and concluded that coping strategies based on focusing were preferable. They defined such focusing strategies as comprising exposure to voice content, responding to that content and modifying underlying beliefs. Focusing now includes metacognitive approaches such as acceptance and mindfulness (Wright 2010).

The key problem is that individuals who experience ongoing distress with auditory hallucinations often activate dysfunctional coping strategies as they try to manage these unpleasant experiences. The strategies used are almost entirely distraction-based techniques aiming to reduce distress and escape from the voices.

Without support and guidance, attempts to use focusing strategies can lead to exacerbation of linked affect (anxiety, anger and shame) and increase unhelpful safety behaviours such as social avoidance, thought suppression or worry (Howard 2012). Instead of helping the voices to diminish, such ineffective coping strategies often perpetuate and exacerbate the maintenance cycle (Fig. 1), as described by Morrison (1998).

Boxes 1-3 give examples of ineffective coping strategies that are commonly used by patients. Distraction techniques such as drowning out the voices (Box 1) can in many ways be more distressing when used incorrectly and can also be distressing

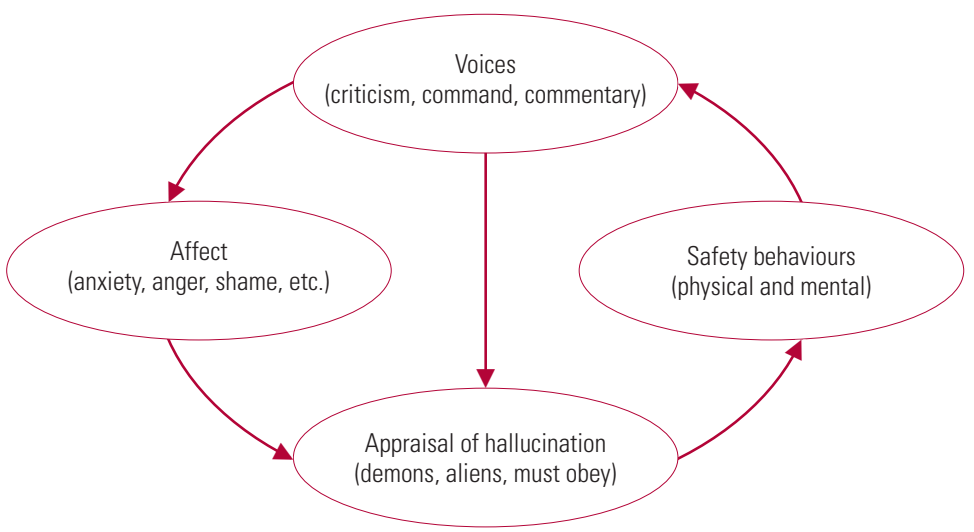

Douglas Turkington is an honorary Professor of Psychosocial Psychiatry; his research interests include cognitive-behavioural therapy (CBT) for schizophrenia, suicide prevention and liaison psychiatry. Latoyah Lebert is a researcher currently working on a randomised controlled trial (RCT) investigating CBT for clozapineresistant psychosis; her research interests include developing CBT techniques for carers of those with psychosis. Helen Spencer is a doctoral researcher investigating the role of formulation in CBT for psychosis; she has previously worked as a researcher on several RCTs, including a trial of CBT for schizophrenia in patients who refuse antipsychotic medication. Correspondence Professor Douglas Turkington, Academic Psychiatry, Wolfson Research Centre, Newcastle General Hospital, Newcastle upon Tyne NE4 6BE, UK. Email: Douglas.Turkington@ntw. nhs.uk
A maintenance model in voice hearing, based on Morrison (1998). 
BOX 1 Ineffective distraction techniques: drowning out the voices

Patients often attempt to overcome critical and abusive voices by using dysfunctional (or unhelpful) distraction techniques. For example, they try to drown out the voices by listening to repetitive sound on headphones. There are three factors here that perpetuate voice hearing:

- the sound is not personally meaningful

- the content is more white noise than structured

- wearing headphones diminishes social contact and reduces the likelihood of activating more efficient coping.

for carers. Shouting back (Box 2) is much more likely to lead to increased stigmatisation, social isolation and hospital admissions. The patient is activating an approach that would appear to them to have face validity: if someone shouts abuse at you, you are likely to shout back. Even certain mindfulness techniques (Box 3) can be counterproductive.

All coping strategies can increase distress if not used appropriately. In essence, the approach recommended here is to identify avoidance or escape-based strategies and then help the patient to move towards efficient distraction (Carter 1996) and, ultimately, focused coping strategies. The choice of strategy should be based on the individual's maintenance formulation, including beliefs about the origin of the voices.

\section{Assessment}

The purpose of assessing how the patient appraises and responds to/deals with their voice hearing

B0X 2 Ineffective focusing approaches: shouting back at the voices

Individuals who hear voices making sexual and/or other allegations often try to manage them (ineffectively) by using a focusing approach. One such strategy is to shout and swear at the voices while pacing up and down the room. Again there are three problems associated with this approach:

- the swearing increases the individual's anger, which in turn raises voice intensity and associated distress, and this serves to maintain the problem

- the pacing and agitation may frighten others and make social contact less likely

- the individual's perceived potential risk to others may result in intervention under the Mental Health Act, leading to increased anger, combined with feelings of stigmatisation and resentment.
BOX 3 Ineffective metacognitive coping strategies: focusing on the voices

Metacognitive coping strategies are rarely naturally activated by patients. Mindfulness shows particular promise as a metacognitive style with strategies that are easily taught and practised. However, some mindfulness exercises (such as the body scan) may be problematic for those patients with unprocessed trauma and a tendency to dissociate. This could activate sadness, shame and anger and exacerbate distress and safety behaviours.

experience is to ask a series of questions to elicit information in a way that helps to illustrate to the patient that some explanations, behaviours and attempts to cope can be directly counterproductive to the goal of recovery.

The assessment might begin with the collaborative creation of a Morrison maintenance model (Fig. 1), identifying triggers, appraisals, affect and safety behaviours. The patient might then complete various informal and formal questionnaires relating to their voice hearing (Box 4). Using the gathered information, the clinician and patient can decide collaboratively whether the current coping strategies are dysfunctional, i.e. are they exacerbating or maintaining the hallucinatory experiences? Basically, if the experience remains distressing and is affecting social functioning, the guided discovery approach will usually lead the patient to the conclusion that what they are doing to cope is perhaps not working very well, or could even be part of the problem. Difficulties with engaging in this process may be related to concern about losing positive voices, fear of commanding voices telling the patient to reject the process, or beliefs that the voices are a core symptom of an illness that cannot be changed. Voices may also be viewed as being very knowledgeable or very powerful. An exploration of the variety of voices that are experienced (and associated beliefs) will allow a focus on the most distressing voices and may involve other cognitive-behavioural strategies to change beliefs about voices.

In different voice hearing contexts, different coping strategies may be needed. A patient who has learned an effective focusing strategy may need to revert to a distraction technique in certain social and emotional situations.

The patient can decide whether or not they would like to learn and attempt a new coping technique. Patients often find it empowering to know that there are many coping strategies that have worked successfully for other voice hearers. They can be given the list of 60 strategies (Wright 2010) recommended by voice hearers, who found 


\section{BOX 4 Assessment of the patient's coping} strategies

The following information can facilitate a collaborative decision concerning the optimum choice of coping strategy:

- the average level of patient distress associated with the voice(s) (scored from 0 to 10: no distress to the highest possible level)

- the patient's level of conviction in their key explanation of the voice(s) for each day (scored from 0 to 10), e.g. belief that the voice is the devil, police, a cyberman

- the level of interference with social functioning associated with the voice(s) (scored from 0 to 10)

- a baseline voice diary over the course of a week, with voice activity, severity, content and linked affect scored from 0 to 10 for the mornings, afternoons and evenings

- score on the Psychotic Symptoms Rating Scales (PSYRATS) auditory hallucinations subscale (Haddock 1999): this gives a sensitive measure of total hallucinatory burden and can be used to measure the utility of a coping strategy on clinical review

- score on the revised Beliefs About Voices Questionnaire (BAVQ-R) (Chadwick 2000): this allows a measure of change in perceived identity, distress, omnipotence and omniscience of the voice as different coping styles are tried

- score on the Brief Core Schema Scales (BCSS) (Fowler 2006): this measure allows core beliefs in schizophrenia to be identified and quantified - these are often echoed by voices (e.g. worthlessness and failure) and can change with effective coping.

them to be of great benefit. It is good practice to give patients a choice of all the strategies on the list. It is also important to point out that the response is idiosyncratic, so what works for one person may well not work for another. If one coping strategy is not effective then there are lots of others to experiment with in the weeks to come. In general, taught distraction is the best place to start (Box 5), as it allows the learning of a coping method without the risk of temporary voice exacerbation. If a focusing strategy is chosen, it should be explained that such strategies may result in a temporary increase in voice severity and distress, before improvement ensues (Kingdon 2005). Instructions and demonstration of the chosen coping strategies can then begin.

\section{Focusing approaches}

The route to recovery with voice hearing is to master an effective personal distraction strategy and, after a number of weeks, to move on to a focusing approach. Focusing is obviously easier if the voice content is less distressing, for example

a running commentary is easier to focus on than a series of personally derogatory commands. It is important to explain how voices are often critical, but that they are basically thoughts that have been externalised and distorted. Often a voice diary will show that a lot of the content is directly related to events in the person's life history and that similar themes and intrusions are present in everybody else's thoughts. Following a successful period of distraction, curiosity as to the nature and content of the voices usually begins to replace the previous terrifying explanations and strong affective and behavioural responses. After all, we have shown the patient that their voices can be controlled at least to some degree to allow a little more space for socially enjoyable activities.

Focusing strategies fall into several categories:

- rational responding

- schema-based techniques

- voice postponement and voice study periods

- writing down voice content

- working with linked imagery

- generating compassion towards the voice

- achieving acceptance in relation to a valued goal

- mindfulness

- attention training.

\section{BOX 5 Examples of effective distraction strategies}

- Listening to music: headphones may be used up to 6 times a day for 20 minutes at a time. The music type is to be negotiated, but should be harmonic, lyrical and slowly paced. The volume should be kept low and other activities are to be encouraged at the same time.

- Playing an instrument: learning or playing a musical instrument is a highly functional coping strategy, as the patient learns to maintain a focus on tone, pitch, rhythm and chords. This approach effectively engages the brain in a creative activity, rather than simply listening to music. The strategy is very effective for some people and in addition may open up the possibility of social contact in terms of playing an instrument with others

- Animals: walking or playing with a dog or other pet is a useful coping strategy as it involves engagement and activity with another living creature, which can have a therapeutic and soothing effect on the individual.

- Watching TV/DVDs: the patient is advised to listen to the sounds of the different voices in the programmes or films (their accent, tone, pitch, etc.) and to aim for non-distressing content (the news is not recommended).

- Breathing exercises: these are useful particularly for individuals whose voices make them anxious. Counting breaths (about 8 to 12 breaths per minute) can have a calming effect. This technique, however, needs to be very clearly described, modelled and practised before the patient attempts it alone.

- Art: this can of course be a full therapy in its own right. For those who are artistically inclined (and can lose themselves in the painting, colours and composition) this is an example of a flow activity that can be an excellent distraction technique.

- Reading: reading activates the speech production and monitoring centres in the brain and for some people is an excellent form of distraction. Again, a flow can be achieved via an enjoyable plot and attending to the different characters in the book. For some voice hearers, switching from internal reading to reading out loud can make a huge difference to the efficacy of the technique. 
The patient practises their chosen strategies each day, scoring them on a 10-point scale to measure their efficacy in reducing the frequency and distress associated with the voice hearing experience. After 7 days, the first coping strategies can be reviewed and either extended for a further 7 days if effective, or abandoned in favour of a different coping technique if there is no evidence of clinical improvement.

\section{Rational responding}

All voices contain distortions, as do most automatic thoughts. The first step is to write the voice content down on a whiteboard or in a diary. This is a focusing breakthrough as the voices are exposed, usually for the first time, to independent analysis. Each voice can be examined to look for its level of veracity. Has the voice got it right? Or is there evidence of distortion such as magnification, labelling, mind-reading error or selective abstraction? On generating a rational response, the patient can speak back to the voice. This is done gently and quietly to 'correct' the distortion in what the voice is saying. These distortions are repeatedly identified and a more rational statement is fed back to the voice, for example:

Voice: 'He's a loser; no one cares about him.' Rational response: 'I am a human being, sometimes I win and sometimes I lose, and I have a girlfriend who cares about me.'

The rational response can then be written on a card or recorded on a CD or mobile phone to remind the patient of the rational responses outside of the clinical session.

\section{Schema-based techniques}

Voices often echo core schemas, such as those linked to trauma or attachment problems. For example:

Voice: 'She's a slut and deserves to burn in Hell.' Rational response: 'I was the victim of an assault when I was a child and the Court ruled that the adult was to blame and not me.'

Here the rational response helps to challenge the underlying trauma-related schema linked to the belief that the child was in some way to blame for the assault.

\section{Voice postponement and voice study periods}

Here the aim is for the patient to learn how to exert some level of control over the voice. The patient is encouraged to focus on the voice and acknowledge it, but only within the limits of the circumscribed 'voice study period', for example between $18.00 \mathrm{~h}$ and $18.30 \mathrm{~h}$ each evening. During this period, the patient actively encourages (and listens to) the voice. They make notes on any possible personal relevance and what can be learned from the voice's attitude and content. These personal reflections are noted in a diary as a homework exercise and attempts are made in the clinical session to try to understand the voices in terms of life history and development. At any other time the voice is dismissed and postponed until the designated voice study period.

\section{Writing down voice content}

Graphotherapy has a considerable evidence base for working effectively with severe mental illness. The very process of writing down internal events allows focusing and has a therapeutic effect. The patient writes a page every day recording what the voices have been saying. It soon becomes very apparent that the content is both negative and repetitive. After 2 weeks of working on this task, one patient threw his diary down and announced to his clinician that the voices were 'boring, same old rubbish every day!'. From this point onwards, he felt more empowered to get on with his usual activities and became less involved and frightened during attentive listening to the voice content.

\section{Working with linked imagery}

When voices are heard there is usually an image activated in the mind. The image is usually congruent with the theme of the voice or its character. Images can of course be captured, changed and replaced with images that are thematically or affectively opposed. One voice hearer, when hearing a voice telling him to attack others, had an image in his mind of a shaven-headed, tattooed, muscular thug. During therapy, he was able to replace this image with the image of someone he respected (Alan Shearer scoring a goal). He found that focusing on this new image in his mind helped him cope with the voice, to be less afraid of it and to feel as though he no longer wished to act on its commands. Patients usually benefit from some brief exercises demonstrating how we can all activate and change images in the mind.

\section{Generating compassion towards the voice}

This can be activated using techniques described by Gilbert (2010). Compassion-focused exercises can be practised, including compassionate selftalk, compassionate meditation, compassionate imagery (for example, a maternal, nurturing image), the creation of a compassionate box (containing, for example, a compassionate letter to the self, or a photograph of someone who cared for them) and compassionate readings. Compassion for the voice can paradoxically be an effective 
focusing approach for coping with voice hearing. A critical voice might say 'You're so rubbish, why not just cut yourself?' A compassionate response might be 'I know that in some way you care about me because otherwise you wouldn't have kept talking to me over so many years'.

\section{Achieving acceptance in relation to a valued goal}

Acceptance is a metacognitive state of mind. It is the opposite of voice suppression, whereby pushing the voices away serves to maintain their recurrence. Using acceptance as a coping style, voice hearing is acknowledged as an aspect of the person's lived experience and no attempts are made to suppress the voices or repeatedly push them away. Acceptance is aided by normalising the experience and educating patients about famous voice hearers (such as Winston Churchill, Ghandi, Joan of Arc and Beethoven), who accepted their hallucinatory experiences and succeeded in their chosen walk of life. The association with creativity can also be emphasised. Acceptance (as defined in acceptance and commitment therapy) is best linked to an identified altruistic valued goal, such as being 'a good grandmother' or 'an honest person' or 'a reliable friend'.

\section{Mindfulness}

This is best taught using experiential exercises (such as the tiger exercise) to demonstrate that the mind never rests and that the individual is actually separated from the activity of the mind. In the tiger exercise, the individual is asked to form a mental image of a tiger and to do nothing with the image except observe it. At the end of the exercise, they will normally report that the tiger has been doing things without their active volition. This teaches the patient how to watch their mind non-judgementally and to allow the voices to come and go (like clouds across the sky) without actively engaging with them. This coping strategy may help to reduce shifts in affect associated with voices, such as anxiety or shame, as well as reducing behavioural components such as escapeor avoidance-based strategies. Instead, the voices are perceived as yet another component of the life of a playful mind. Mindfulness is practised at various times throughout the day, for example in mindful breathing, mindful walking and mindful tasting. Mindfulness training as a religious discipline is not recommended in the early stages of recovery.

\section{Attention training}

Attention training has been described by Wells (1994) in relation to the control of worry. To disrupt the cognitive attentional state that serves to maintain troublesome voices, the individual is trained to practise shifting their attention to different auditory stimuli (for example, the ticking of a clock, the sound of traffic, a radio or birdsong).

Repeated practice of attentional shifts improves the person's ability to move attention away from their voices, allowing them to try less emotionally engaged coping strategies.

\section{Conclusions}

The Morrison maintenance model allows clinicians to explain to patients that voice hearing is common and understandable, and can be worked with successfully. Giving up ineffective coping strategies to practise new, more effective strategies can be challenging for some patients, but offering them examples of successful voice management along with a list of 60 coping strategies (Wright 2010) can engender hope and change. The route to recovery may be enhanced by first identifying ineffective coping strategies, then teaching the patient effective distraction techniques and, finally, focusing techniques that will ultimately diminish the voice hearing experience, leaving the person with voice-free periods and a marked reduction in distress.

\section{References}

Carter DM, Mackinnon A, Copolov DL (1996) Patients' strategies for coping with auditory hallucinations. Journal of Mental and Nervous Disease, 184: 159-64.

Chadwick P, Lees S, Birchwood M (2000) The revised Beliefs About Voices Questionnaire (BAVQ-R). British Journal of Psychiatry, 177: 229-32.

Fowler D, Freeman D, Smith B, et al (2006) The Brief Core Schema Scales (BCSS): psychometric properties and associations with paranoia and grandiosity in non-clinical and psychosis samples. Psychological Medicine, 36: 749-59.

Gilbert P (2010) Compassion Focused Therapy: Distinctive Features. Routledge.

Haddock G, Bentall RP, Slade PD (1996) Psychological treatment of auditory hallucinations: focusing or distraction? In Cognitive-Behavioural Interventions with Psychotic Disorders (eds G Haddock, PD Slade): 45-71. Routledge.

Haddock G, Morrison AP, Hopkins R, et al (1998) Individual cognitivebehavioural interventions in early psychosis. British Journal of Psychiatry, 172 (suppl 33): s101-6.

Haddock G, McCarron J, Tarrier N, et al (1999) Scales to measure dimensions of hallucinations and delusions: the Psychotic Symptoms Rating Scales (PSYRATS). Psychological Medicine, 29: 879-89.

Howard A, Forsyth A, Spencer H, et al (2012) Do voice hearers naturally use focusing and metacognitive coping techniques? Psychosis, 5: 119-6.

Kingdon DG, Turkington D (2005) Cognitive Therapy of Schizophrenia. Guilford Press.

Morrison AP (1998) A cognitive analysis of auditory hallucinations: are voices to schizophrenia what bodily sensations are to panic? Behavioural and Cognitive Psychotherapy, 26: 289-302.

Wells A (1994) Attention and the control of worry. In Worrying: Perspectives on Theory, Assessment and Treatment (eds GCL Davey, F Tallis): 91-114. Wiley.

Wright J, Sudak DM, Turkington D, et al (2010) High-Yield CognitiveBehavior Therapy for Brief Sessions: An Illustrated Guide. American Psychiatric Publishing.
MCO answers

1 e 2 e 3 b 4 b 5 e 


\section{MCOs}

Select the single best option for each question stem

1 Which of the following is an effective coping strategy for voices?

a Shouting back

b Listening to loud rock music

c Using cannabis

d Staying alone all day

e Writing the voices down in a diary.

2 Which of the following is not a key component of Morrison's voice maintenance model?

a Auditory hallucinations

b Safety behaviours

c Strong emotions

d An extreme appraisal

e Attention training.
3 Which of the following is not a focusing approach?

a Mindfulness

b Voice suppression

c Imagery modification

d Voice postponement and voice study periods

e Rational responding.

4 A patient has been practising shifting his attention to the sound of a wind chime in his room; after 7 days there has been no improvement in his distress. Which step should be taken next?

a Cut the wind chime down and bin it

b Introduce two other different sounds to practise with in sequence

c Get him to practise ignoring the voice

d Increase his medication

e Shift his attention to watching horror DVDs.
5 A young man who is being bombarded with commanding voices refuses to leave the house. Which of the following is least likely to help him cope?

a In collaboration with his therapist, constructing a Morrison maintenance model of the voice hearing experience

b Learning to play a musical instrument for 30 minutes a day, with the help of a teacher

c Spending 30 minutes a day writing down the content of his voices

d Listening to an audio-book of his choice and using a voice diary to monitor his voices

e Repeatedly forcing the voices away. 